\title{
Transcription factors in airway diseases
}

\author{
Peter J Barnes \\ Section of airway diseases, National Heart $₹$ Lung Institute, Imperial College, London, UK
}

\begin{abstract}
Transcription factors regulate the expression of multiple inflammatory genes and play a pivotal role in chronic inflammatory diseases, such as asthma and chronic obstructive pulmonary disease (COPD). Prominent transcription factors in airway diseases include nuclear factor- $\kappa$ B (NF- $\kappa$ B) and activator protein-1 (AP-1), which together regulate the expression of multiple inflammatory proteins. Glucocorticoids activate glucocorticoid receptors (GR), which act as transcription factors and inhibit transcription induced by NF- $\kappa$ B and AP-1. Activation of genes involves hyperacetylation of core histones to open up the chromatin structure to initiate transcription. GR recruit histone deacetylase-2 (HDAC2) to the activated inflammatory gene to switch off transcription. In COPD, there is a marked reduction in HDAC2 expression, resulting in glucocorticoid resistance. Therapeutic implications, which may be generalized to all inflammatory diseases, are discussed. Laboratory Investigation (2006) 86, 867-872. doi:10.1038/labinvest.3700456; published online 24 July 2006
\end{abstract}

Keywords: NF- $\kappa$ B; AP-1; NF-AT; glucocorticoid receptor; asthma; COPD

In asthma and chronic obstructive pulmonary disease (COPD) there is increased expression of multiple inflammatory proteins in the respiratory tract, including cytokines, adhesion molecules, inflammatory enzymes and inflammatory receptors. Most of these inflammatory proteins are regulated at the level of gene transcription. Changes in gene transcription are regulated by transcription factors, which are proteins that bind to DNA. This suggests that transcription factors may play a key role in the pathophysiology of chronic airway diseases, since they regulate the increased gene expression that may underlie the chronic inflammatory process. They may also be a target for novel anti-inflammatory treatments.

\section{What are transcription factors?}

Transcription factors are proteins that bind to regulatory sequences, usually in the $5^{\prime}$ upstream promoter region of target genes, to increase (or occasionally decrease) the rate of gene transcription (Figure 1). This may result increased (or sometimes) decreased protein synthesis and altered cellular function. Transcription factors may be activated via surface receptors through phosphorylation by several kinase cascades, or may be activated by ligands (such as glucocorticoids). Transcription factors may therefore convert transient signals at

Correspondence: Professor PJ Barnes, DM, DSc, FRCP, Section of airway diseases, National Heart \& Lung Institute, Imperial College, Dovehouse Street, London SW3 6LY, UK.

Received 25 May 2006; revised 26 May 2006; accepted 21 June 2006; published online 24 July 2006 the cell surface into long-term changes in gene transcription, thus acting as 'nuclear messengers'.

Several families of transcription factors exist and members of each family may share structural characteristics. Many transcription factors are common to several cell types (ubiquitous), whereas others are more cell-specific and determine the phenotypic characteristics of a cell. Transcription factors, by determining which genes are expressed in a particular cell, therefore, determine why a liver cell is different from a smooth muscle cell, for example.

While transcription factors play a key role in the long-term regulation of cell function, growth and differentiation, they may also play a pivotal role in chronic inflammatory diseases. ${ }^{1}$ There is increasing evidence that transcription factors are critical in the expression of cytokine genes and may therefore be involved in inflammatory and immune diseases. Indeed, it is now recognized that some therapies used in the treatment of these diseases, such a glucocorticoids, may function through interaction with transcription factors. It remains a possibility that abnormal functioning of transcription factors may determine disease severity and responsiveness to treatment. Of particular importance is the demonstration that transcription factors may interact with each other, resulting in inhibition, or enhancement, of transcriptional activity.

There is growing information about the role of transcription factors inflammatory diseases. One of the most important concepts to have emerged is that transcription factors may interact with other transcription factors. This then allows cross-talk between different signal transduction pathways at the level of gene expression. Indeed, it is the 


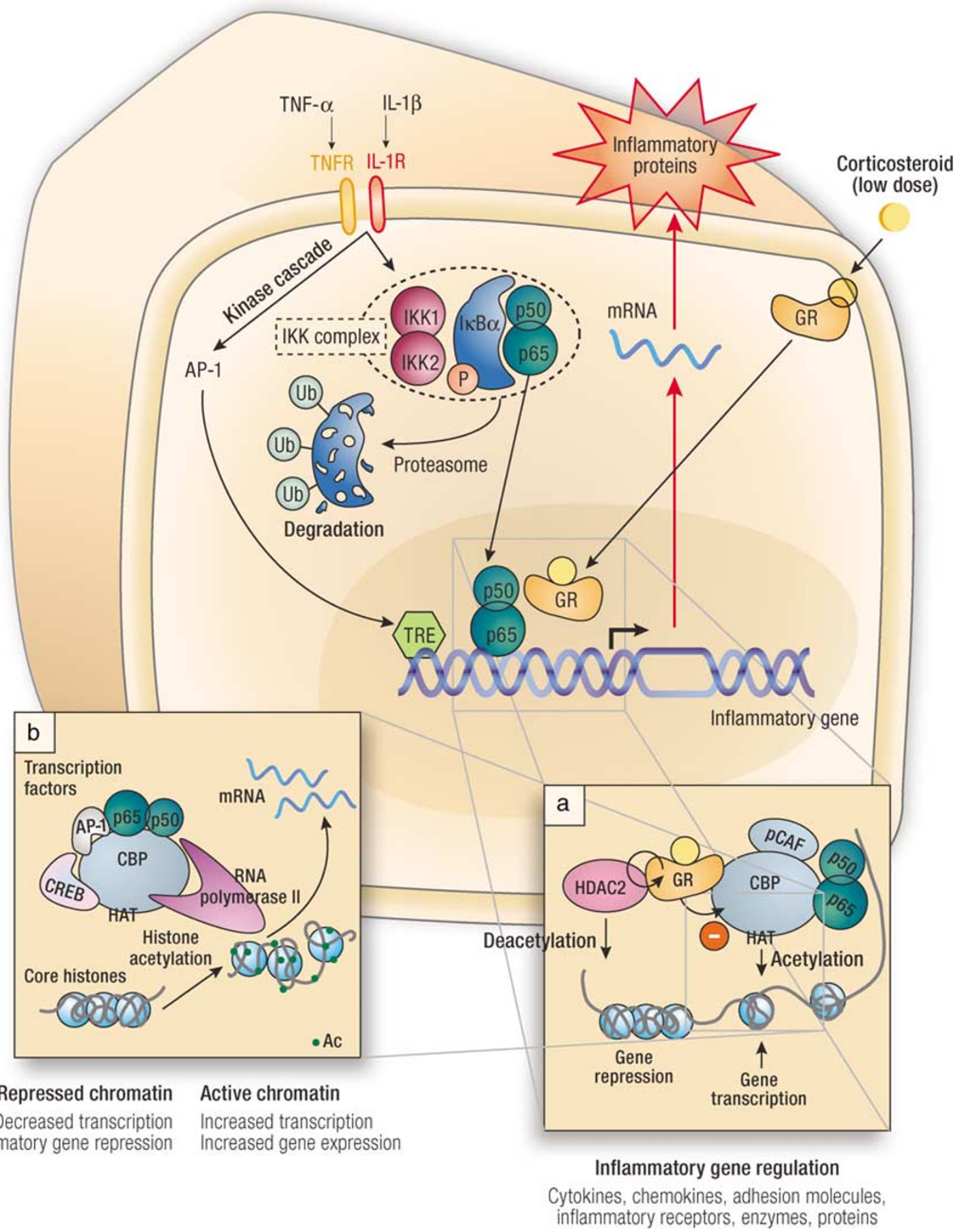

Figure 1 Transcription factors. TF, such as NF- $\kappa$ B and AP-1, are activated in the cytoplasm by extracellular stimuli that activate signal transduction pathways (kinase cascades). In the case of NF- $\kappa \mathrm{B}$, external stimuli such as TNF- $\alpha$ and IL- $1 \beta$ signal through specific receptors to activate the IKK2. In turn, IKK2 phosphorylates inhibitor of NF- $\kappa \mathrm{B}(\mathrm{I}-\kappa \mathrm{B} \alpha)$, leading to its ubiquitination and subsequent degradation by the proteasome. This frees up the p65 and p50 subunits of NF- $\kappa \mathrm{B}$, which translocate to the nucleus. Intranuclear NF- $\kappa \mathrm{B}$ then binds to $\kappa \mathrm{B}$ recognition sites in the $5^{\prime}$-promoter (upstream) region of inflammatory genes, resulting in increased gene transcription, messenger RNA, and synthesis of inflammatory proteins. Inset a: Upon translocation to the nucleus, the p50-p65 heterodimer NF- $\kappa \mathrm{B}$ binds to specific $\kappa \mathrm{B}$ recognition sites and also to coactivators, such as CREB-binding protein (CBP) or p300/CBP-activating factor (PCAF), which have intrinsic histone acetyltransferase (HAT) activity. This results in acetylation of lysines in core histone-4, resulting in increased expression of genes encoding inflammatory proteins, such as granulocyte-macrophage colony-stimulating factor (GM-CSF). Glucocorticoid receptors (GR) after activation by corticosteroids translocate to the nucleus and bind to coactivators to inhibit HAT activity directly and specifically recruit histone deacetylase-2 (HDAC2), which reverses histone acetylation leading in suppression of inflammatory genes. Inset b: Coactivators, such as CREB-binding protein (CBP) have intrinsic histone acetyltransferase (HAT) activity, resulting in opening up to the chromatin structure, which allows binding of RNA polymerase II and initiation of gene transcription. Several transcription factors interact with CBP, including CREB (cyclic AMP response element binding protein), NF- $\kappa$ B (nuclear factor$\kappa \mathrm{B})$ and AP-1 (activator protein-1). 
interaction of transcription factors that may give them different properties in different cell types, as the presence of other transcription factors will profoundly influence the effect exerted by a particular factor on gene expression. Interaction between transcription factors is particularly relevant to the action of drugs, such as glucocorticoids and cyclosporin A, which activate transcription factors that subsequently modulate other transcription factors.

Many important questions about transcription factors remain to be answered. They appear to regulate different inflammatory genes and may even by activated by different stimuli in different cells. This may be explained by the interaction of several transcription factors, which may differ between cell types. Gene array analysis and proteomics will be invaluable in sorting out different panels of genes between different cells.

\section{Key transcription factors involved in airway diseases}

\section{Nuclear Factor-Kappa B}

Nuclear factor-kappa B (NF- $\kappa$ B) plays an important role in the regulation of cell activity. ${ }^{2}$ It is an important transcriptional regulator of several inducible genes, including inducible nitric oxide synthase (iNOS), the inducible form of cyclooxygenase (COX-2), chemotactic cytokines (chemokines) such as interleukin (IL)-8 and eotaxin, and adhesion molecules such as ICAM-1 and VCAM-1 that play a key role in inflammatory cell recruitment (Figure 1). NF- $\kappa \mathrm{B}$ is present in the cytoplasm in an inactive form, complexed to an inhibitory protein, $\mathrm{I} \kappa \mathrm{B}$. Upon cell activation, $\mathrm{I} \kappa \mathrm{B}$ kinase-2 (IKK2) phosphorylates $\mathrm{I} \kappa \mathrm{B}$, and after ubiquitination, the proteasome rapidly degrades $\mathrm{I} \kappa \mathrm{B}$, resulting in its dissociation from NF- $\kappa \mathrm{B}$. NF- $\kappa \mathrm{B}$ itself is made up of two subunits, a protein of $65 \mathrm{kDa}$ (p65 or Rel A), and a protein of $50 \mathrm{kDa}$ (p50), both of which are members of the Rel family. ${ }^{3}$ Free NF- $\kappa \mathrm{B}$ localizes to the nucleus, where it binds to specific $\kappa \mathrm{B}$ recognition elements on the promoter region of target inflammatory genes. NF- $\kappa \mathrm{B}$ may be activated by cytokines, such as tumor necrosis factor-A (TNF $\alpha)$ and protein kinase $\mathrm{C}$ (PKC), which has been demonstrated in many inflammatory cells. ${ }^{4} \mathrm{NF}-\kappa \mathrm{B}$ also activates the transcription of one form of $\mathrm{I} \kappa \mathrm{B}, \mathrm{I} \kappa \mathrm{B}-\alpha$, which then acts as a autoregulatory feedback inhibitor, thus limiting the activation of NF- $\kappa \mathrm{B}$. NF- $\kappa \mathrm{B}$ is also activated by oxidants, such as hydrogen peroxide $\left(\mathrm{H}_{2} \mathrm{O}_{2}\right)$ and may thus function as an oxidative-stress responsive transcription factor. This may be relevant in chronic inflammation when oxidants, such as superoxide anions are generated by inflammatory cells and in asthma where inhalation of environmental oxidants, such as ozone, may amplify inflammation. Viral infections may also activate $\mathrm{NF}-\kappa \mathrm{B}$ and this may account for the worsening of many inflammatory diseases, such as asthma by intercurrent viral rhinovirus infection. By contrast, glucocorticoids inhibit NF- $\kappa \mathrm{B}$ activation, and this appears to be via interaction of the glucocorticoid receptor (GR) with $\mathrm{NF}-\kappa \mathrm{B}$ through binding to coactivators and corepressors. Thus, steroids inhibit expression of inducible nitric oxide synthase (iNOS), cyclo-oxygenase-2 (COX-2), chemokines and adhesion molecules, at least in part, by blocking the activation of NF- $\kappa \mathrm{B}$. NF- $\kappa \mathrm{B}$ is increased in the airways of asthmatic patients, but is not reduced by glucocorticoid therapy, indicating that GR inhibits $\mathrm{NF}-\kappa \mathrm{B}$ indirectly (see below). ${ }^{5}$

\section{Activator Protein-1}

Activator protein-1 (AP-1) is a heterodimer of Fos and Jun oncoproteins and was originally described by binding to the TPA (tetradecanoylphorbol-13acetate) response element (TRE). It was found to be responsible for the transcriptional activation of various genes that were activated by phorbol esters (such as TPA, now better known as PMA) via activation of protein kinase $\mathrm{C}$ (PKC). It is now apparent that AP-1 is a collection of related transcription factors belonging to the Fos (c-Fos, FosB, Fra1, Fra2) and Jun (c-Jun, JunB, JunD) families, which dimerize in various combinations through a region known as a leucine zipper. AP-1 may be activated via PKC and by various cytokines, including $\mathrm{TNF} \alpha$ and interleukin (IL)-1 $\beta$, via several types of protein tyrosine kinase (PTK) and mitogenactivated protein (MAP) kinase, which themselves activate a cascade of intracellular kinases. ${ }^{6}$ Certain signals rapidly increase the transcription of the fos gene, resulting in increased synthesis of Fos protein. Other signals lead to activation of kinases that phosphorylate c-Jun, resulting in increased activation. Specific Jun and Fos kinases are now recognized, and may play an important role in the regulation of cellular responsiveness to cytokine signals. Conversely a Jun phosphatase counteracts the activation of AP-1, and a deficiency of this enzyme might lead to amplification of chronic inflammation. The activated GR interacts directly and indirectly with activated AP-1, and this may be an important action of steroids to inhibit cytokinemediated inflammatory responses.

\section{Glucocorticoid Receptors}

GRs themselves are transcription factors and regulate the transcription of several steroid-responsive target genes. ${ }^{7}$ Glucocorticoids bind to GR in the cytoplasm, resulting in rapid nuclear localization of GR. GR binds to positive glucocorticoid response elements (GREs), resulting in increased gene transcription (as in the case of $\beta_{2}$-adrenergic receptors) or to negative GREs (as in the case of some cytokines). There is now compelling evidence that many of the anti-inflammatory effects of steroids may be due to a direct or indirect interaction 
between GR and transcription factors, such as AP-1 and $\mathrm{NF}-\kappa \mathrm{B}$, that have been activated by cytokines and that regulate the expression of several inducible genes. By contrast, when there is an active cytokinemediated inflammatory process these transcription factors may inactivate GR, resulting in reduced steroid responsiveness. In the case of $\mathrm{NF}-\kappa \mathrm{B}$, glucocorticoids may increase the synthesis of $\mathrm{I} \kappa \mathrm{B}$ in some cell types, thus providing an additional mechanism for inhibiting NF- $\kappa \mathrm{B}$ activation.

A small proportion of patients with inflammatory diseases such as asthma and rheumatoid arthritis are steroid-resistant and fail to respond to even high doses of oral steroids. ${ }^{8}$ This defect is also seen in mononuclear cells isolated from these patients. There is little evidence for a reduced number or affinity of GR, but there is a marked impairment of GRE binding after exposure of mononuclear cells to steroids in vitro. This is associated with a reduction in the number of activated GR available for binding. In the same patients there is an increased activation of AP-1, suggesting that the AP-1 may consume activated GR, thereby blocking the anti-inflammatory actions of steroids. ${ }^{9}$ The underlying defect in AP-1 is likely to be an abnormality in one of the enzymes involved in AP-1 activation. Glucocorticoid resistance is very common in COPD (see below).

\section{Nuclear Factor of Activated T-Cells}

Nuclear factor of activated T-cells (NF-AT) is a good example of a cell-specific transcription factor, as it is largely confined to T-lymphocytes. NF-AT is of key importance in the regulation of the expression of IL2 and probably other T-cell derived cytokines, such as IL-4 and IL-5. Activation of T cells results in activation of calcineurin, which activates a preformed cytoplasmic NF-AT (NF-ATp). It is now clear that AP1 forms a transcriptional complex with NF-AT (and is the nuclear NF-AT previously identified) to increase IL-2 gene expression. This may be inhibited by cyclosporin A and tacrolimus (FK 506) which inhibit calcineurin, or by steroids which inhibit AP- $1 .{ }^{10}$ Both of these calcineurin inhibitors have been shown to have anti-inflammatory effects in asthma.

\section{Histone acetylation and deacetylation}

A very active area of research is modification of core histones by acetylation, phosphorylation and methylation within the chromatin structure (epigenetic), resulting in chromatin remodeling and profound effects on gene expression. Gene expression is regulated by acetylation of core histones which open up the chromatin structure to allow transcription factors and RNA polymerase to bind to DNA, thus initiating transcription. ${ }^{11}$ Gene expression is regulated by various coactivator molecules that all have intrinsic histone acetyltransferase activity. In asthma and COPD expression of inflammatory genes is mainly regulated by increased acetylation of histone- 4 induced by the binding of NF- $\kappa \mathrm{B}$ or AP-1. ${ }^{1}$ Histone acetylation is reversed by histone deacetylases (HDAC). There are 11 HDAC isoenzymes that deacetylate histones within the nucleus and specific HDACs appear to be differentially regulated and to regulate different groups of genes. This expression of inflammatory genes is determined by a balance between histone acetylation, which activates transcription, and deacetylation, which switches off transcription.

We have demonstrated that recruitment of HDAC2 by activated GR to the hyperacetylated coactivators reverses the increase in gene transcription induced by NF- $\kappa \mathrm{B} .{ }^{12}$ HDAC2 activity and expression are reduced in alveolar macrophages in peripheral lung, bronchial biopsies and alveolar macrophages from COPD patients and this is correlated with disease severity and with increased gene expression of IL-8. ${ }^{13}$ HDAC2 is necessary to deacetylate the acetylated GR before it can inhibit NF- $\kappa$ B. ${ }^{14}$ This also accounts for the glucocorticoid resistance in COPD patients as switching off of inflammatory genes is impaired.

The reasons for the reduction in HDAC, particularly HDAC2, in COPD are not yet completely understood. However, there is increasing evidence that this may be due to inactivation of the enzyme of oxidative and nitrative stress ${ }^{15}$ (Figure 2). Oxidative and nitrative stress lead to the formation of peroxynitrite, which nitrates tyrosine residues on certain proteins. HDAC2, but not other isoforms of HDAC, shows increased tyrosine nitration in macrophages and peripheral lung of COPD patients and this is correlated with increased expression of IL-8. Nitration of proteins such as HDAC2 leads to their ubiquitination, which marks them for degradation by the proteasome. The high level of oxidative/nitrative stress in COPD lungs may therefore result in increased tyrosine nitration and impaired HDAC2 function and reduction in expression, which thereby leads to increased expression of inflammatory genes and impaired responses to glucocorticoids. ${ }^{16}$ Cigarette smoking also reduces HDAC2 activity and this may explain why asthmatic patients who smoke have a markedly reduced response to glucocorticoids. ${ }^{17}$

\section{Therapeutic implications}

The study of transcription factors has provided important insights into inflammatory diseases, such as asthma and COPD, accounting for increased expression of multiple inflammatory genes and how thay can be suppressed by a single drug, such as glucocorticoids. This has important clinical applications, leading to the development of transcription factor inhibitors. While direct inhibitors have been difficult to discover, the kinase pathways that activate transcription factors have been successfully targeted. Relatively selective IKK2 have now 


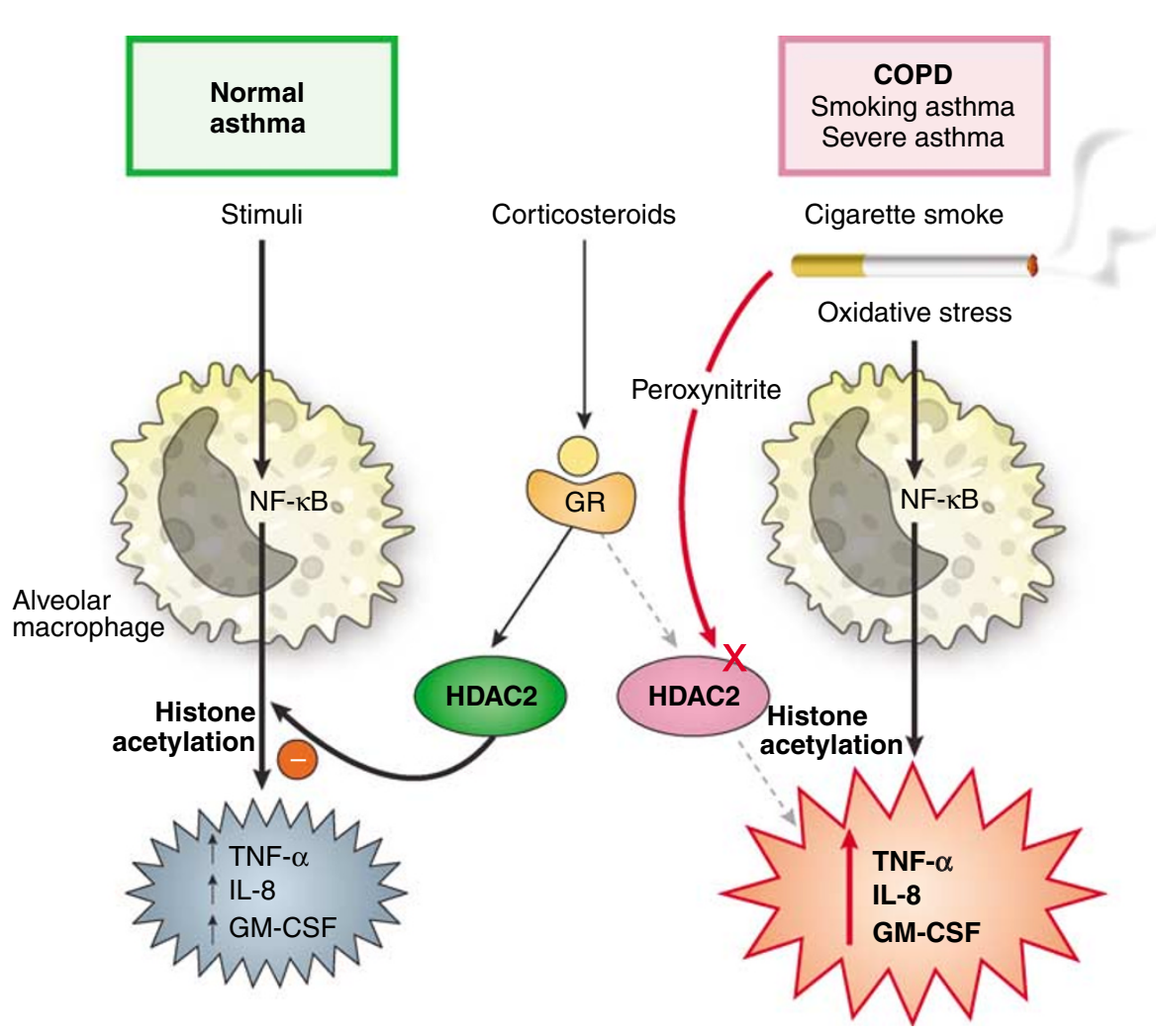
of genes encoding inflammatory proteins, such as TNF- $\alpha$ and IL-8. Corticosteroids reverse this by binding to GR and recruiting HDAC2. This reverses the histone acetylation induced by NF- $\kappa$ B and switches off the activated inflammatory genes. In COPD patients cigarette smoke activates macrophages, as in normal subjects, but oxidative stress (acting through the formation of peroxynitrite) impairs the activity of HDAC2. This amplifies the inflammatory response to NF- $\kappa$ B activation, but also reduces the anti-inflammatory effect of corticosteroids as HDAC2 is now unable to reverse histone acetylation.

been developed which inhibit NF- $\kappa \mathrm{B}$-mediated gene expression and these drugs are moving into clinical trials. ${ }^{18}$ Similarly Jun kinase inhibitors are also in clinical development. The discovery of a key role for HDAC2 in switching off inflammation has prompted a search for activator compounds. To date the only such compound is theophylline, which is also able to reverse glucocorticoid resistance in COPD. ${ }^{19}$ In the future, novel HDAC activators may be discovered and the signaling pathways that regulate HDAC are discovered. ${ }^{20}$ This holds the prospect for many new drugs in the future that would be effective in airway diseases, but also in other common chronic inflammatory diseases, such as rheumatoid arthritis and inflammatory bowel disease.

\section{Future perspectives}

Understanding transcription factors has given us extraordinary insights into the underling mechanisms of chronic inflammatory diseases, such as asthma and COPD. We now need to dissect the differential response between different cell types and learn more about how transcription factors interact. The development of relatively selective inhibitors of signal transduction pathways that activate transcription factors now allows the role of specific transcription factors, such as NF- $\kappa \mathrm{B}$ and AP-1, to be studied in experimental animals in vivo, and hopefully eventually to clinical studies, if such approaches prove to be nontoxic. The new research on the role of histone acetylation in regulation of inflammatory genes and their suppression by glucocorticoids is particularly exciting as it opens up entirely new areas of research. The demonstration that reduced HDAC2 activity causes glucocorticoid resistance raises the possibility of novel HDAC2 activators in the future to restore glucocorticoid sensitivity so that low and safe doses are effective. Theophylline may be a starting point for the discovery of such drugs. ${ }^{20}$ This would be clinically useful in many inflammatory diseases when the disease is severe, particularly COPD which is the commonest inflammatory disease in the world that is glucocorticoid-resistant and is the common disease in the USA which shows a rising mortality. This new approach could transform the management of chronic inflammatory diseases in the future. Exciting times are ahead and much research now needs to be done in different inflammatory diseases! 


\section{Acknowledgements}

Supported by various grants from the Wellcome trust, Medical Research Council, British Lung Foundation and Asthma UK.

\section{References}

1 Barnes PJ, Adcock IM. Transcription factors and asthma. Eur Respir J 1998;12:221-234.

2 Barnes PJ, Karin M. Nuclear factor- $\kappa$ B: a pivotal transcription factor in chronic inflammatory diseases. New Engl J Med 1997;336:1066-1071.

3 Karin M, Ben-Neriah Y. Phosphorylation meets ubiquitination: the control of NF- $\kappa$ B activity. Annu Rev Immunol 2000;18:621-663.

4 Catley MC, Cambridge LM, Nasuhara Y, et al. Inhibitors of protein kinase $\mathrm{C}$ prevent activated transcription: Role of events downstream of NF- $\kappa$ B DNA binding. J Biol Chem 2004;279:18457-18466.

5 Hart L, Lim S, Adcock I, et al. Effects of inhaled corticosteroids in expression and activations of nuclear factor-kB (NF-kB) in asthma. Eur Respir J 1998;12:115S.

6 Karin M, Liu ZG, Zandi E. AP-1 function and regulation. Curr Opin Cell Biol 1997;9:240-246.

7 Barnes PJ. How corticosteroids control inflammation. Br J Pharmacol 2006;148:245-254.

8 Adcock IM, Lane SJ. Corticosteroid-insensitive asthma: molecular mechanisms. J Endocrinol 2003;178:347-355.

9 Adcock IM, Lane SJ, Brown CA, et al. Abnormal glucocorticoid receptor/AP-1 interaction in steroid resistant asthma. J Exp Med 1995;182:1951-1958.

10 McCaffrey PG, Perrino BA, Soderling TR, et al. NFATp, a T lymphocyte DNA-binding protein that is a target for calcineurin and immunosuppressive drugs. J Biol Chem 1993;268:3747-3752.
11 Adcock IM, Ford P, Barnes PJ, et al. Epigenetics and airways disease. Respir Res 2006;7:21.

12 Ito K, Barnes PJ, Adcock IM. Glucocorticoid receptor recruitment of histone deacetylase 2 inhibits IL- $1 \beta$ induced histone $\mathrm{H} 4$ acetylation on lysines 8 and 12 . Mol Cell Biol 2000;20:6891-6903.

13 Ito $\mathrm{K}$, Ito $\mathrm{M}$, Elliott WM, et al. Decreased histone deacetylase activity in chronic obstructive pulmonary disease. New Engl J Med 2005;352:1967-1976.

14 Ito K, Yamamura S, Essilfie-Quaye S, et al. Histone deacetylase 2-mediated deacetylation of the glucocorticoid receptor enables NF- $\kappa \mathrm{B}$ suppression. J Exp Med 2006;203:7-13.

15 Barnes PJ, Ito K, Adcock IM. A mechanism of corticosteroid resistance in COPD: inactivation of histone deacetylase. Lancet 2004;363:731-733.

16 Ito $\mathrm{K}$, Tomita $\mathrm{T}$, Barnes PJ, et al. Oxidative stress reduces histone deacetylase (HDAC)2 activity and enhances IL-8 gene expression: role of tyrosine nitration. Biochem Biophys Res Commun 2004;315: 240-245.

17 Thomson NC, Spears M. The influence of smoking on the treatment response in patients with asthma. Curr Opin Allergy Clin Immunol 2005;5:57-63.

18 Catley MC, Sukkar MB, Chung KF, et al. Validation of the anti-inflammatory properties of small molecule IKK2 inhibitors by comparison to adenoviral-mediated delivery of dominant negative IKK1 and IKK2 in human airways smooth muscle. Mol Pharmacol 2006 (in press).

19 Cosio BG, Tsaprouni L, Ito $\mathrm{K}$, et al. Theophylline restores histone deacetylase activity and steroid responses in COPD macrophages. J Exp Med 2004;200: 689-695.

20 Barnes PJ. Targeting histone deacetylase 2 in chronic obstructive pulmonary disease treatment. Expert Opin Ther Targets 2005;9:1111-1121. 